\title{
Predicting Virus Evolution: The Relationship between Genetic Robustness and Evolvability of Thermotolerance
}

\author{
C.B. Ogbunugafor, R.C. McBride, and P.E. Turner \\ Department of Ecology and Evolutionary Biology, Yale University, New Haven, Connecticut 06520-8106 \\ Correspondence: paul.turner@yale.edu
}

\begin{abstract}
Evolutionary biologists often seek to infer historical patterns of relatedness among organisms using phylogenetic methods and to gauge the evolutionary processes that determine variation among individuals in extant populations. But relatively less effort is devoted to making evolutionary biology a truly predictive science, where future evolutionary events are precisely foreseen. Accurate predictions of evolvability would be particularly useful in the evolution of infectious diseases, such as the ability to preemptively address the challenge of pathogens newly emerging in humans and other host populations. Experimental evolution of microbes allows the possibility to rigorously test hypotheses regarding pathogen evolvability. Here, we review how genetic robustness was a useful predictor in gauging which variants of RNA virus $\phi 6$ should evolve faster in a novel high-temperature environment. We also present new data on the relative survival of robust and brittle viruses across elevated temperatures and durations of ultraviolet exposure, to infer a possible mechanism for robustness. Our work suggests that virus adaptability in a new environment can be predicted given knowledge of virus canalization in the face of mutational input. These results hint that accurate predictions of virus evolvability are a realistic possibility, at least under circumstances of adaptive thermotolerance.
\end{abstract}

As a naturalist, Charles Darwin was understandably fascinated by the species diversity visible in the natural world. However, he understood little of the invisible realm of genes - the units of inheritance that are passed across generations and contribute to the phenotypic variation evident in biological populations. Yet Darwin showed remarkable insight when formulating his ideas on evolution via natural selection, as a process whereby the environment determines which variants in a population are favored to contribute their traits to succeeding generations, leading to microevolutionary changes over short timescales. Furthermore, Darwin perceptively understood that natural selection is a process that can also primarily account for nature's vast biodiversity-resulting from macroevolution occurring over long timescales - which Darwin described as "endless forms most wonderful" (Darwin 1859).

Many modern-day evolutionary biologists are concerned with elucidating patterns of extant diversity, seeking to understand how descent with modification from common ancestry accounts for Earth's myriad forms that have evolved since life arose billions of years ago. In contrast, other evolutionary biologists are largely concerned with the processes of evolutionary change that underlie these patterns, examining how mechanisms such as selection and drift can lead to altered phenotypes and genotypes across relatively few generations. Both camps of evolutionary biologists have increasingly powerful and sophisticated approaches for studying patterns and processes of evolution, allowing more accurate glimpses into the mysterious "black boxes" of past and ongoing evolutionary events (see, e.g., Lenski et al. 2003; Thornton et al. 2003; Vrba and DeGusta 2004; Weinreich et al. 2006).
Arguably, evolutionary biology mostly concerns scrutiny of past and present events, rather than forthcoming events, i.e., evolutionary biologists tend to resist the temptation to study and predict how evolution will play out in the future. Such predictive efforts are necessarily more difficult than retrospective analyses, because foretelling the future is inherently less precise than resolving the past. For this reason, relatively less energy has been placed into developing evolutionary biology into a truly predictive science. Of course, like other scientists, evolutionary biologists often make explicit predictions, referred to as hypothesis testing. But there is a distinct difference between formulating a hypothesis that concerns events that have already occurred and formulating a hypothesis that predicts events yet to occur.

\section{EVOLVABILITY STUDIED USING MATHEMATICAL THEORY AND BIOLOGICAL EXPERIMENTS}

Mathematical studies in evolutionary biology are sometimes future-minded. For example, John Maynard Smith (1982) famously popularized evolutionary game theory, a discipline that often seeks to mathematically determine whether a population can evolve an unbeatable strategy when dealing with competition for finite resources, such as food, mates, or nesting sites. If this evolved strategy is truly unbeatable, it is deemed an evolutionarily stable strategy (ESS) whereby all members of the population should adopt the strategy, preventing the population's invasion by any competing strategy in the future. However, evolutionary game theory usefully acknowledges that the strategy may fail to be an ESS should the environment change unex- 
pectedly, because the rules of engagement may summarily change as well. Because the future may be uncertain, evolutionary games such as tit for tat allow players to change their strategies through time, depending on each other's actions and similarly variable conditions (Turner and Chao 2003; Cressman et al. 2004; Wagner 2006)

Many other examples of future-minded mathematical theory exist in evolutionary biology, centering on the topic of evolvability. Here, the general goal is to predict which genotypes should be advantaged to give rise to evolutionarily successful populations, even when precise environmental changes are difficult to foresee. Evolvability can be defined as the ability to adapt through natural selection (Wagner and Altenberg 1996; Kirschner and Gerhart 1998; Brookfield 2009). Mathematical treatments of evolvability are highly useful, but like all mathematical models in biology, they necessarily must work within confined frameworks reflecting a subset of real-world conditions, i.e., all relevant biological parameters cannot possibly be incorporated into a mathematical model or else the model quickly becomes mathematically intractable and too complicated to be useful. A helpful goal is to identify biological factors deemed most important for making the evolvability prediction and then use these to build the model.

The preparation of flu vaccines is one example of the need to apply evolvability thinking to a practical problem. How can we best predict which variants of influenza virus will be most prevalent during the next flu season, given that stocks of the flu vaccine must be prepared and distributed to medical workers long before the flu season actually strikes? Mathematical and genomic approaches developed for evolutionary phylogenetics have proven to be highly useful for this vital medical task (Bush et al. 1999; Koelle et al. 2006; Coburn et al. 2009). Essentially, one can look retrospectively to determine which virus variants have been recent evolutionary successes and then apply an algorithm to deduce which of these viruses will be prevalent in the near future to warrant choosing them as vaccine targets. Of course, it is well known that mass-produced vaccines are not always highly effective in protecting against the flu, and entirely new variants of influenza virus may emerge unexpectedly to threaten human health (e.g., H1N1 variants identified during a 2009 outbreak in Mexico). These observations aptly demonstrate our sometimes limited capability to accurately predict the course of future evolutionary events. Do such findings indicate that studies of evolvability and other topics in evolutionary prediction should remain locked into the realm of mathematical theory?

Not necessarily. Experimental evolution is one empirical discipline in evolutionary biology where researchers are willing to let their science be forward-looking. Experimental evolution is the study of evolution in action, often involving biological populations placed in controlled laboratory environments where researchers scrutinize phenotypic and molecular changes occurring across generations. Because experimental evolution allows the study of evolutionary change as it unfolds, such studies span the past (a founding ancestor) through the present (evolved descendants). Furthermore, if the study system can be archived (stored under suspended animation), genotypes from dif- ferent evolutionary time periods may be directly compared, such as through head-to-head competition. Microbes such as viruses, bacteria, and yeast often allow this intense scrutiny because they may be stored indefinitely under ultralow temperature and then revived at a later time. Thus, experimental evolution of microbes allows evolving populations to march forward in time, and the future conveniently becomes the present as researchers examine changes taking place across this completely accessible "fossil record."

Here, we review our studies in experimental evolution of viruses, emphasizing how this work can be used to study evolvability. We deemed that it was useful to first identify whether viral genomes evolved to differ in genetic robustness: relative ability to maintain a constant phenotype despite random mutational input (de Visser et al. 2003). This link between phenotype and genotype is crucial for evolution, because natural selection is fueled by phenotypically expressed genetic variation. As we discuss below, robustness is a trait that dictates the translation of genotype to phenotype, and knowledge of virus robustness may be used to predict whether viruses should be relatively advantaged in their evolvability when encountering future environmental challenges.

\section{ROBUSTNESS DESCRIBES THE LINK BETWEEN GENOTYPE AND PHENOTYPE}

Evolution is defined as the change in genetic makeup of a population through time. This change can be driven by the process of natural selection, which leads indirectly to changes in gene frequencies by acting on the phenotypes that genes produce. Thus, the translation of phenotype from genotype is vital to the natural selection process. Robustness and brittleness are terms used for measuring and describing the relative accuracy of this genotype-to-phenotype translation (Fig. 1). If a mutation changes a phenotype, we consider the gene-or, more broadly, the genome - to be relatively nonrobust (brittle) against mutational input. However, if a genome changes but its phenotype remains unaffected, the genome is considered genetically robust. Because other types of robustness are of interest, especially environmental robustness that describes whether a phenotype persists when an environment changes, it is crucial when examining robustness to define the specific phenotype and perturbation being measured and discussed. As we describe below, to better understand how natural selection shapes the adaptive trajectory of a population, it would be useful to know the average effectiveness of genotype-to-phenotype translation (genetic robustness) for individuals in the population when adaptive challenges are encountered.

Despite a long-standing interest in evolution of robustness and its associated mathematics, evolutionary biologists have generated few empirical data on this subject. Some empirical studies have allowed organisms to evolve for long periods in a constant environment (see, e.g., Lenski and Travisano 1994), and one might expect that these lineages would be ideal candidates to study evolution of genetic robustness; at evolutionary equilibrium — mutation-selecti- 

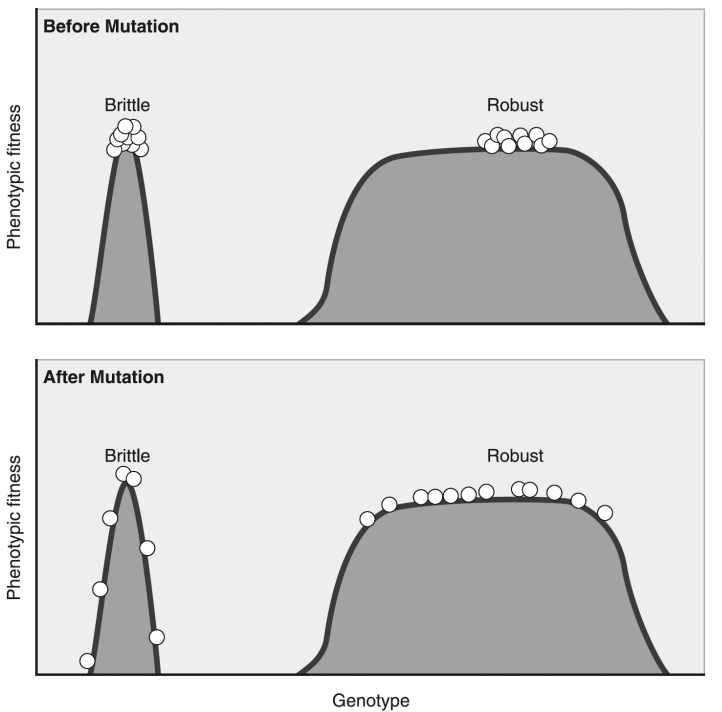

Figure 1. Brittle and robust organisms can be defined by their fitness response to mutational change, using the metaphor of fitness landscapes. Fitness is vertical height on the landscape. Mutation causes genotypes to move away from their original position on the horizontal axis. After mutation, brittle individuals experience large changes in fitness as they are "pushed off" the narrow fitness peak. Because robust individuals reside on flatter portions of the landscape, they are phenotypically buffered against mutational change. (Modified, with permission, from Wilke and Adami 2003 [@ Elsevier].)

on balance - any mutation entering the population should be either neutral or deleterious, perhaps causing strong selection for genetic robustness that protects the phenotype against mutations. However, theory suggests that such selection may be weak or even absent (Wagner et al. 1997) due to opposing effects of stabilizing selection that increases the fitness effect of the mutations present in a population while simultaneously reducing the frequency of mutants in the equilibrium population. In contrast, because spontaneous mutations are believed typically to be deleterious, selection favoring evolution of robustness should be especially strong if mutation rates are elevated, even when populations are away from equilibrium (Wilke et al. 2001).

Some convincing data on evolution of robustness stem from studies looking at virtual organisms, namely, selfreplicating computer programs that change randomly and thus have the potential to evolve. According to one such study (Wilke et al. 2001), elevated mutation rates can cause robust genotypes to be selectively favored over their brittle counterparts, even though robustness against mutations coincided with lower reproductive fitness. Thus, the fittest gave way to the "flattest" with selection favoring those variants having the greatest phenotypic constancy and residing on flat regions of the fitness landscape (Fig. 1) (Wilke et al. 2001). Other studies successfully examined robustness by following changes in proteins that are evolved in vitro (Bloom et al. 2007; Bloom and Arnold 2009).

Microbial populations are logical candidates for examining the evolution of robustness due to their rapid generation times and large population sizes. Similar to digital organism experiments, elevated mutation rates should be a key prerequisite for microbial populations to adapt by altering their genetic (mutational) robustness (Wilke et al. 2001). RNA viruses seem to be particularly appropriate for examining genetic robustness because their mutation rates generally exceed those of other organisms, including DNA viruses, by at least one order of magnitude (Drake et al. 1998).

\section{DEMONSTRATING EVOLUTION OF ROBUSTNESS IN RNA VIRUSES}

A handful of experimental evolution studies with RNA viruses have been used to show that genetic robustness can increase or decrease, depending on the particulars of the selective environment. In experiments with RNA viroids of plants and with vesicular stomatitis virus, an RNA virus that infects mammals and insect vectors, populations evolved in treatments causing elevated mutation rates were observed to undergo selection for increased genetic robustness (Codoñer et al. 2006; Sanjuán et al. 2007). These results echo the conclusions drawn from digital organism experiments where robustness evolves in response to mutation pressure (Wilke et al. 2001).

In our experiments with the RNA virus $\phi 6$, we examined a different selection pressure where evolved changes in robustness were expected (Montville et al. 2005; see also Turner and Chao 1998). We used a single genotype of the lytic RNA bacteriophage (phage) $\phi 6$ to found six populations. Three of these populations were allowed to adapt to growth on the bacterium Pseudomonas syringae pathovar phaseolicola at very low multiplicity of infection (moi; ratio of viruses to bacteria); when moi $<<1.0$, the vast majority of infection events are expected to be single phage particles infecting individual host cells to produce viral progeny (Turner et al. 1999). In parallel, the remaining three populations evolved at a high multiplicity (moi $\approx 5$ ), where two to three phage particles on average should coinfect an individual host cell (Turner et al. 1999). This experimental evolution study lasted for 300 phage generations (60 days, five generations per day) (Fig. 2).

A key difference between the two experimental treatments was that high-multiplicity viruses could experience complementation, a mechanism that can effectively allow viruses to experience robustness against mutations (Froissart et al. 2004). In theory, adaptive robustness assumes that phenotypic expression results solely from the underlying genotype. However, this assumption should not hold true for viruses that can experience complementation, because virus genotypes typically have no ownership rights over the proteins encoded by their genomes. For example, during coinfection, a low-fitness viral genotype can phenotypically profit from intracellular proteins made by a coinfecting virus of higher fitness, causing the low-fitness virus to be overrepresented among the viral progeny exiting the cell (Froissart et al. 2004). Therefore, complementation during coinfection can automatically cause viral phenotypes to be beneficially buffered against mutations. By analogy, viral complementation has a role similar to that of gene duplication and diploidy, genetic mechanisms that may provide robustness in cellular or multicellular organisms. 


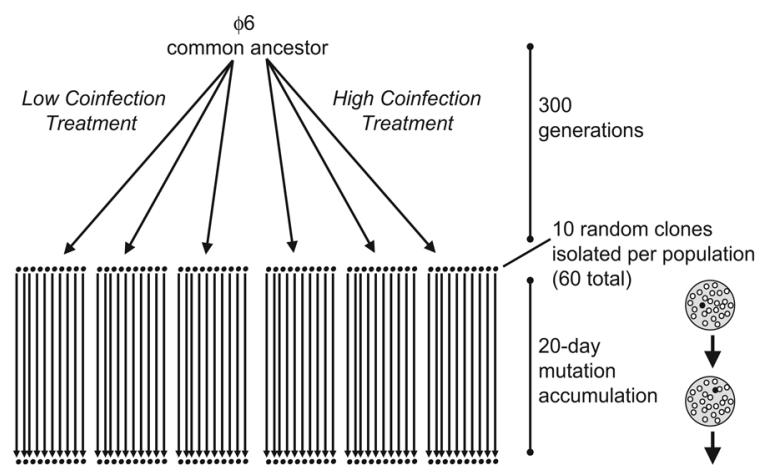

Figure 2. Design of an experiment where a wild-type phage $\phi 6$ ancestor founded three lineages evolved under a low level of coinfection, and three lineages evolved at high coinfection. After 60 days (300 generations), 10 clones were isolated from each population and used to found lineages subjected to mutation accumulation (MA). The MA lineages were then analyzed to see whether prior ecological history (low vs. high coinfection) affected robustness: maintenance of constant phenotype (fitness) despite mutational change. (Modified from McBride et al. 2008.)

Because complementation buffers mutational effects, it offers a built-in robustness mechanism for populations of phage $\phi 6$ that experience coinfection. By this logic, conditions fostering complementation (and hence, coinfection) may weaken selection for phage $\phi 6$ genomes to maintain their individual-level robustness, because coinfection provides mutational buffering. We therefore predicted that the degree of coinfection-high multiplicity versus low multiplicity-should influence evolution of robustness in phage $\phi 6$ populations (Montville et al. 2005). More specifically, we hypothesized that selection for robustness should be greatly relaxed in the phage $\phi 6$ populations that evolved for 300 generations under high levels of coinfection. If true, this would mean that the high-multiplicity populations should be dominated by $\phi 6$ genotypes that are relatively less robust to mutations.

To test the idea, we isolated 10 phage clones at random from each of the three low- and high-multiplicity populations (60 clones total). We then used each clone to found an independent population. These 60 populations were then subjected to a mutation accumulation experiment, where a population experiences an extreme daily bottleneck of a single virus particle (Fig. 2). To do so, we plated a dilution of each phage $\phi 6$ population on a host lawn and the next day chose a plaque at random for the next round of population growth. Thus, the population is propagated via daily plaque-to-plaque transfers imposing an extremely small population size, allowing the effects of genetic drift to be relatively more important than natural selection in dictating evolutionary change. This propagation scheme enables a phage population to accumulate nonlethal mutations at random (Chao 1990; Burch and Chao 2004). Because random mutations are expected to be deleterious on average, mean fitness of a virus population is expected to decline through time as random mutations accumulate.
The 60 phage populations were subjected to 20 consecutive days of extreme bottlenecking, providing the opportunity for $\sim 1.3$ mutations to fix in each population, based on an estimated rate of 0.067 mutations per generation in phage $\phi 6$ (Burch and Chao 2004). To examine how the amassed mutations affected phenotypic fitness ( $W$, relative growth rate on the host bacteria), we measured $\log _{10} W$ of each prebottleneck and postbottleneck population. The difference between these two values, $\Delta \log _{10} W$, revealed the sensitivity of the population to phenotypic effects of the accumulated mutation(s) (Fig. 3). Support for the hypothesis would be that the 30 populations initiated by clones that were historically evolved under high multiplicity (frequent coinfection) would show greater variance in $\Delta \log _{10} W$ values, owing to weakened selection for them to maintain robustness as an individual trait. Additional support would come from a greater mean magnitude of $\Delta \log _{10} W$ values for the high-multiplicity populations, indicating that they suffered a greater drop in fitness on average, because they are less able to withstand the deleterious effects of mutations. These hypothesized outcomes are depicted in Figure 3.

The data supported the general predictions (Fig. 4), confirming that selection to maintain mutational robustness is weaker with viral coinfections - viruses that were historically evolved under low-coinfection conditions were measurably advantaged in robustness relative to their high-coinfection-evolved counterparts, i.e., results demonstrated that viruses evolved under high moi showed relatively greater mean magnitude and variance in the fitness changes generated by addition of random mutations (Fig. 4). From these results, we concluded that the lowcoinfection populations could henceforth be defined as relatively genetically robust, whereas the high-coinfection populations were considered to be relatively brittle.

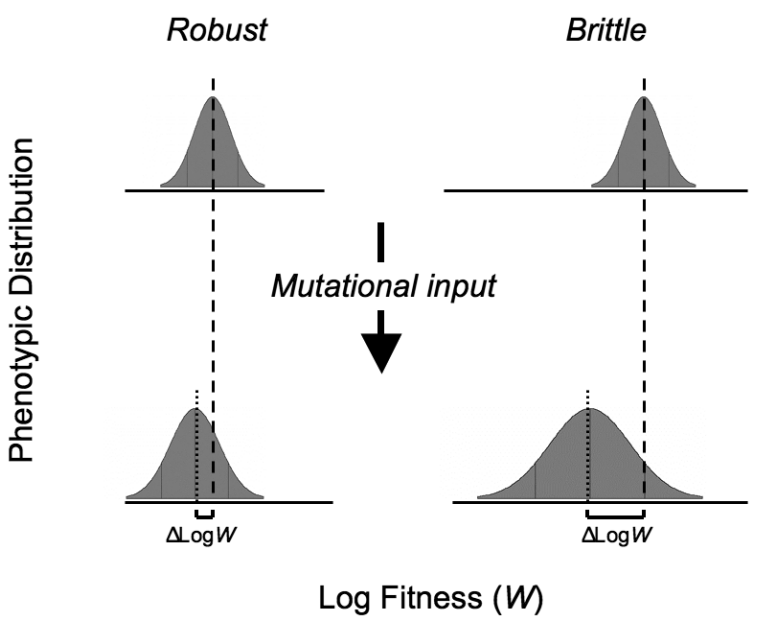

Figure 3. Hypothetical diagram showing how relative robustness of organisms may be revealed through measurements of fitness change following mutational input. Because the population on the right presents greater variance in fitness and lower mean fitness following mutational change, it can be defined as relatively less robust (i.e., brittle). 


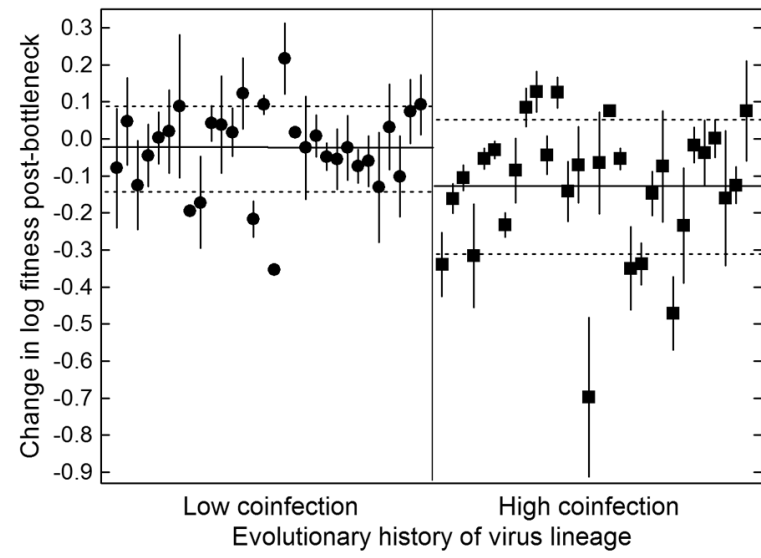

Figure 4. Phage $\phi 6$ strains that evolved under low coinfection are more robust than those evolved under high coinfection, owing to their lower variance in fitness and higher mean fitness following mutation accumulation. Each point is the mean change in $\log _{10}$ fitness resulting from mutation accumulation, for an independent lineage founded by a virus clone previously evolved under a low level of coinfection (closed circles) or a high level of coinfection (closed squares). (Horizontal lines) Grand means among lineages within a treatment, (dashed lines) one standard deviation away from the mean. (Modified from Montville et al. 2005.)

So far, the exact molecular mechanism responsible for robustness in phage $\phi 6$ has not been determined. But some clues regarding the mechanism stem from additional sets of experiments examining the relationship between genetic robustness and evolvability and between genetic robustness and generalized environmental robustness.

\section{HOW DOES ROBUSTNESS IN THE CURRENT ENVIRONMENT IMPACT ADAPTATION SHOULD THE ENVIRONMENT CHANGE?}

On the one hand, constancy in the face of environmental and mutational changes provides obvious benefits to an organism during growth and reproduction. Robustness buffers organisms against such perturbations, affording constancy in terms of cellular function, development, and offspring production, i.e., robustness provides reliability in the very currencies by which natural selection judges phenotypes. On the other hand, rigidity in the face of change may pose problems. For example, if organisms are steadfast under environmental change, how can they possibly adapt to new conditions? Because natural selection acts on phenotypic variation, robustness that buffers this variation could impede evolution.

These conflicting necessities force organisms to strike a balance between withstanding some changes and maintaining an ability to adapt to new circumstances. This compromise is the balance between robustness and evolvability, the capacity to adapt. It is conceivable that some variants are selectively favored over others in striking this balance, such that these favored genotypes are more likely to give rise to progeny that dominate the population should a novel environmental challenge be encountered. Thus, by examining this balancing act, we may learn whether evolvability can itself evolve. Furthermore, we can explore the intriguing - and contentious - idea that natural selection shapes evolution itself (Wagner and Altenberg 1996; Kirschner and Gerhart 1998; Wagner 2005)

Where does one begin in addressing issues of such high stakes in evolutionary biology? Some prior theoretical and empirical studies in biology offer clues; evolvability has been explored in disciplines ranging from protein biophysics to yeast genetics (Bloom et al. 2004, 2006; Tanay et al. 2005; Tokuriki and Tawfik 2009). These varying contexts often share a common theme: Prior evolution of protein-folding stability seems to be beneficial for future evolutionary innovation. When faced with new environmental challenges, robust proteins (those that maintain their folding stability despite underlying genetic changes) seem to be better capable of experiencing mutations that permit innovation without compromising proper folding. Thus, populations composed of individuals with relatively robust proteins are expected to have an adaptive advantage in novel environments.

Although many environmental stressors can harm organisms by negatively impacting protein folding, heat shock is an especially direct proxy for protein stability, as several studies in protein thermostability have contributed to our broader understanding of protein structure and stability (Kumar et al. 2001; Razvi and Scholtz 2006). This notion is supported by the observation that microbial species thriving in extremely high-temperature environments typically possess proteins with unusually stable folding patterns that facilitate growth and survival under thermodynamic stress at elevated temperatures (Huber and Stetter 2001; Sterner and Liebl 2001; England et al. 2003). The widespread existence of heat shock protein chaperones in biological systems offers further evidence that protein stability under heat shock is a target of natural selection (e.g., in yeast species) (Craig et al. 1993; Parsell et al. 1993) .

For these reasons, we can infer that phage $\phi 6$ genotypes which differ in genetic robustness may also differ in survival under heat shock. In addition, evolution of the ability to tolerate survival under high temperatures provides a logical choice for interrogating whether relatively robust genotypes of the virus are advantaged when founding populations that need to adapt under novel conditions. Below, we summarize how we used phage $\phi 6$ as a biological system to examine the potential positive relationship between genetic robustness and evolvability.

\section{EXAMINING THE LINK BETWEEN GENETIC ROBUSTNESS AND EVOLVABILITY IN RNA VIRUS $\phi 6$}

We have used experiments with phage $\phi 6$ to address whether robustness promotes or hinders evolvability (McBride et al. 2008; see also McBride and Turner 2008). In the wild, phage $\phi 6$ and other members of the Cystoviridae virus family seem to specifically attack plant pathogenic Pseudomonas species that colonize plant surfaces (Silander et al. 2005). Thus, phage $\phi 6$ is typically cultured in the laboratory at the benign temperature of $25^{\circ} \mathrm{C}$. We observed that when bacteria-free lysates of wild-type $\phi 6$ 
were exposed to 5-min heat shock at temperatures above $40^{\circ} \mathrm{C}$, increasingly fewer virus particles remained viableable to successfully infect the typical host $P$. syringae pathovar phaseolicola (McBride et al. 2008). At heat shock of $45^{\circ} \mathrm{C}, \sim 80 \%$ of wild-type virus particles became nonviable. Presumably, the $45^{\circ} \mathrm{C}$ heat shock severely damages viral proteins, such as the $\mathrm{P} 3$ attachment protein of the virus that is needed for cell attachment during the early stages of infection.

From the collection of 60 virus clones that were isolated from populations which experienced 300 generations of viral evolution under high versus low moi (Montville et al. 2005), we chose a subset of 24 clones to study the relationship between robustness and evolvability. These 24 clones (12 robust, 12 brittle) were first examined for their survival under $45^{\circ} \mathrm{C}$ heat shock. Results showed that the two groups of viruses were similarly sensitive in terms of average percent survival $(\% S)$, with mean survival for both robust and brittle genotypes of only 14\% (McBride et al. 2008). Because both groups of $\phi 6$ viruses were earlier subjected to 300 generations of evolution with daily incubation at $25^{\circ} \mathrm{C}$, we did not expect the robust and brittle clones to differ in their sensitivity to high temperature. However, this information confirmed that we could conduct an evolution experiment examining whether robustness enhanced or suppressed evolvability under heat shock conditions, without biasing in favor of one group of the viruses.

Our preliminary experiments also addressed other potential confounders. In particular, we examined whether the two groups of virus populations (robust and brittle) differed in mutation rate. Regardless of robustness differences, a higher mutation rate could foster more rapid adaptation in a new environment because a population can more easily access rare beneficial mutations. Such a difference could then lead to spurious conclusions regarding the link between robustness and evolvability in a new habitat. However, two separate methods for gauging spontaneous frequencies of viral mutants did not suggest that robust and brittle viruses consistently differed in mutation rate (McBride et al. 2008). A second potential confounder was whether one set of viruses was better fit under low-moi conditions, because we intended to gauge evolvability in the absence of coinfection. The robust and brittle viruses had historically evolved under low- versus high-moi conditions, respectively (Turner and Chao 1998). But our assays showed that after 300 generations of virus evolution, the fitness of robust and brittle clones was equivalent at low moi (McBride et al. 2008), even though performance trade-offs across these moi environments were evident earlier in the evolutionary process (Turner and Chao 1999). Because these trade-offs had eroded by the time of our evolvability study, we deemed that any observed differences in the rate of adaptation to $45^{\circ} \mathrm{C}$ heat shock would be due to differences in intrinsic evolvability of the viruses.

To conduct the experimental evolution under heat shock, we used each of the 12 robust and 12 brittle clones to found an independent test population. These 24 test populations were allowed to undergo 50 generations (10 days) of evolution, where each population experienced growth on $P$. phaseolicola at $25^{\circ} \mathrm{C}$, with periodic (every fifth generation) exposure to $45^{\circ} \mathrm{C}$ heat shock (Fig. 5, top). At the end of the study, we measured mean $\% S$ at $45^{\circ} \mathrm{C}$ for each founding clone and its derived endpoint population. These replicated measures were used to estimate mean $\Delta \% S$, the average change in percent survival after 50 generations of selection to resist damaging heat shock. The results showed that the populations founded by robust genotypes were more evolvable (Fig. 5, bottom). From these data, we concluded that robustness promoted evolv-
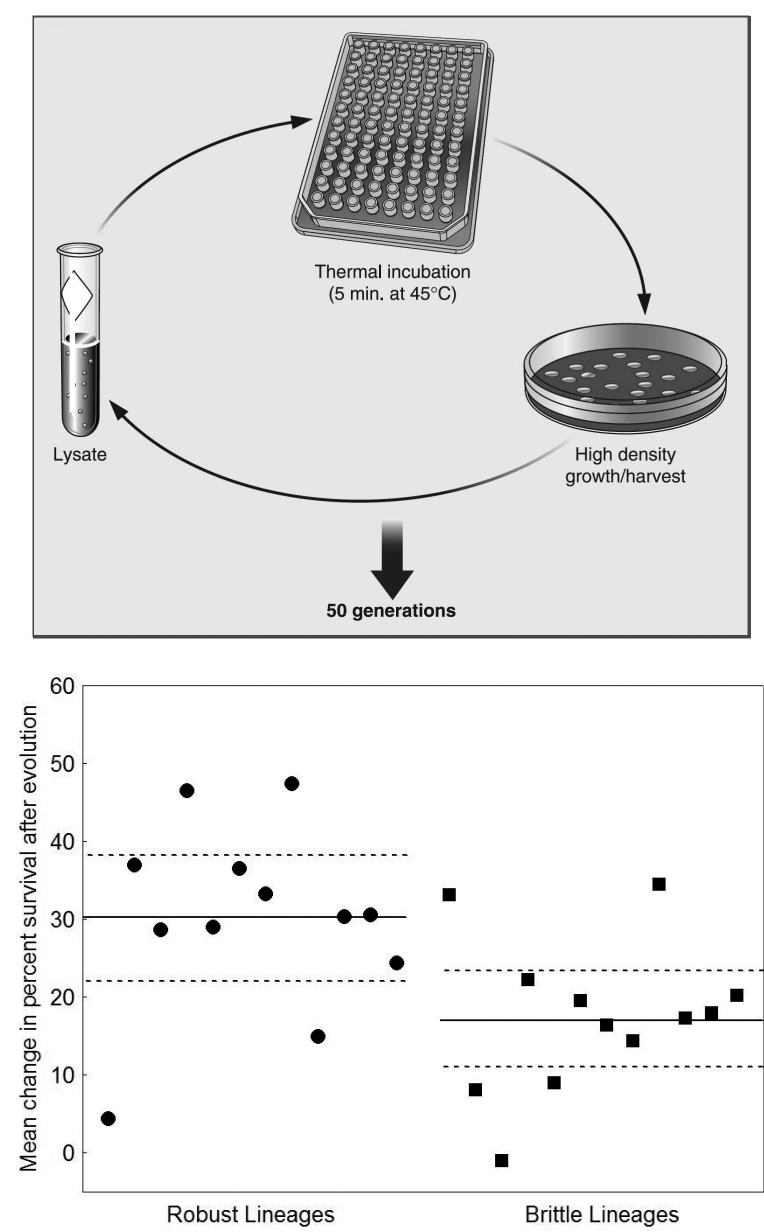

Figure 5. (Top) Design for an evolution experiment where phage $\phi 6$ lineages were selected to withstand damaging effects of heat shock. A phage lysate was exposed to $45^{\circ} \mathrm{C}$ incubation for $5 \mathrm{~min}$, and a dilution of the surviving progeny was plated on a lawn of $P$. phaseolicola bacteria. Overnight plaque formation at $25^{\circ} \mathrm{C}$ corresponded to five generations of phage evolution. The plaques were then harvested, and the process was repeated for 10 days, equivalent to 50 phage generations. (Bottom) Mean change in $\% S$ after selection with heat shock $\left(45^{\circ} \mathrm{C}\right)$ is greater for virus lineages founded by 12 robust strains (closed circles), relative to lineages initiated by 12 brittle strains (closed squares). All populations were subjected to 10 days (50 generations) of periodic heat shocks. (Solid line) Grand mean for the group, (dashed lines) 95\% confidence intervals. (Top, Modified, with permission, from McBride and Turner 2008 [ $₫$ ASM]; Bottom, modified from McBride et al. 2008.) 
ability in phage $\phi 6$, at least when viruses underwent adaptation to resist the damaging effects of survival at high temperature. Below, we present new data relating to a potential mechanism for robustness in phage $\phi 6$.

\section{NEW DATA ON A POSSIBLE UNDERLYING MECHANISM FOR VIRUS ROBUSTNESS}

Data from in vitro protein studies suggest that less-sensitive (relatively robust) proteins are more likely to maintain their function in a new environment where innovation is needed (Bloom et al. 2006; Ortlund et al. 2007). A similar mechanism may explain differences in robustness among phage $\phi 6$ strains and may account for the link between robustness and evolvability of thermotolerance (McBride et al. 2008), i.e., robust strains may contain proteins that tend to tolerate mutations while maintaining proper folding. Under heat shock selection, evolution of greater protein thermostability would be advantageous. We infer that robust viruses featured one or more proteins that were better able to maintain proper folding while allowing the input of spontaneous mutation(s), which led to exploration of novel thermostable genotypes. This beneficial combination would explain the increased ability of the robust viruses to adapt under heat stress. In contrast, the brittle viruses were perhaps constrained in their ability to adapt because their proteins encountered mutations that increased thermostability while compromising the ability to produce viral progeny efficiently. Better thermotolerance of the robust viruses would support existing arguments for the connection between robustness and thermostability (Bornberg-Bauer 1997; Bornberg-Bauer and Chan 1999).

We performed additional experiments to further examine the protein stability mechanism that links phage $\phi 6$ robustness to evolvability. Empirical support for the mechanism would be increased survivability of robust strains at lessextreme temperatures than the $45^{\circ} \mathrm{C}$ selective environment, where survival of robust and brittle viruses was shown to be equally poor (McBride et al. 2008). Thus, we hypothesized that robust viruses should show better average survival under moderate-temperature heat shock, relative to brittle viruses. To test this idea, we compared survival of 12 phage clones (six robust, six brittle) that constitute a subset of the strains used to found populations in our evolvability experiment. In particular, we chose six phage clones from the group of viruses historically evolved under low coinfection, previously described as relatively robust and better evolvable under $45^{\circ} \mathrm{C}$ heat shock: L1.7, L1.9, L2.4, L2.6, L3.2, and L3.8. The remaining six phage clones were from the group evolved under high coinfection, described as comparatively brittle and less adaptable to heat shock: H1.1, H1.3, H2.10, H2.4, H3.5, and H3.9.

Each of the 12 phage strains was used to obtain a hightiter lysate, which was diluted to a concentration of $\sim 2.5 \mathrm{x}$ $10^{3}$ plaque-forming units (pfu) $/ \mathrm{ml}$. A $30-\mu \mathrm{L}$ volume from each lysate ( $750 \mathrm{pfu})$ was placed in a sterile PCR (polymerase chain reaction) tube and immediately sampled onto a lawn of the $P$. phaseolicola host; plaques that formed during overnight incubation were used to accurately estimate initial virus titer $\left(N_{\mathrm{i}}\right)$. The PCR tube was then incubated for $5 \mathrm{~min}$ in an Eppendorf thermocycler, preheated to an assay temperature of $25^{\circ} \mathrm{C}, 37.5^{\circ} \mathrm{C}, 40^{\circ} \mathrm{C}$, $42.5^{\circ} \mathrm{C}$, or $45^{\circ} \mathrm{C}$. After this temperature shock, $10 \mu \mathrm{L}$ of the test lysate was sampled onto a host lawn of bacteria to estimate final virus titer $\left(N_{\mathrm{f}}\right) . \% S$ was calculated using the formula $\% S=\left(N_{\mathrm{f}} / N_{\mathrm{i}}\right) \times 100$. Three independent replicate measurements of $\% S$ were obtained for each virus clone at each of the five assay temperatures, and we calculated the mean $\% S$ for each clone at a given temperature. Grand mean $\% S$ values for the two groups of viruses (robust, brittle) at a given temperature were obtained by averaging the clone means within each group.

The results showed that mean survival of robust and brittle viruses was not statistically different at the standard growth temperature of $25^{\circ} \mathrm{C}$ ( $t$-test with $t=0.067, d f$ $=10, P=0.947)$. Figure 6 plots the reaction norm for the grand mean $\% S$ of robust viruses and of brittle viruses, across the four elevated heat shock environments $\left(37.5^{\circ} \mathrm{C}, 40^{\circ} \mathrm{C}, 42.5^{\circ} \mathrm{C}, 45^{\circ} \mathrm{C}\right)$. Visual inspection suggests that the reaction norm of the brittle viruses is linear, whereas that of the robust viruses is curvilinear, concave downward. A statistical analysis confirmed that the data for the brittle viruses were best described by a simple linear regression $(P=0.007)$. In contrast, a quadratic regression $(P=0.071)$ provided a better fit to the data for the robust viruses than did a linear regression $(P=0.083)$. However, we noted that both of these fits to the data for the robust viruses were marginally significant, indicating variation in $\% S$ among the robust strains (data not shown). We also conducted a Kaplan-Meier survival estimator to analyze differences in the survival functions of the two virus groups. A log-rank comparison test showed that the robust viruses had a survival function that significantly differed from that of the brittle viruses $(\alpha=0.05$; $P=0.015)$. From these analyses, we concluded that the robust viruses showed better average survival at moderately warm temperatures. This outcome is consistent with

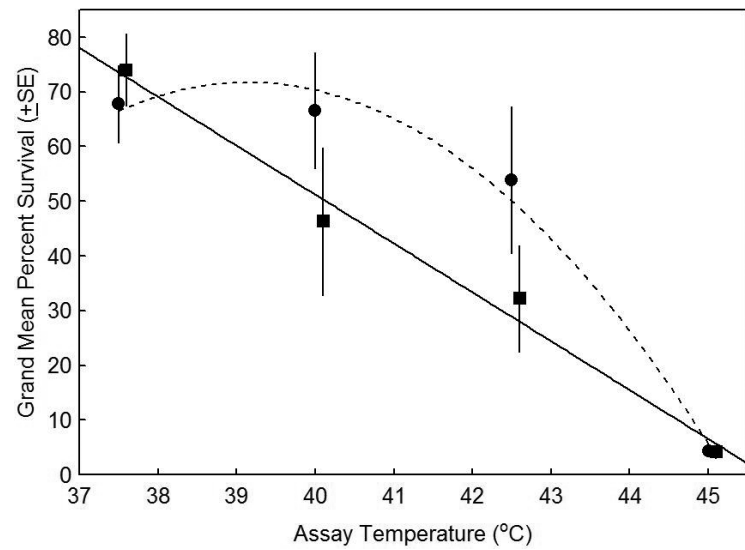

Figure 6. Strains of phage $\phi 6$ that differ in genetic robustness also differ in survival at moderately high temperatures. Brittle (closed circles, dashed line) and robust (closed squares, solid line) viruses were exposed to heat shock at a range of temperatures, and the two groups of viruses differed in survival as a function of temperature. Data points are offset for clarity. 
the protein stability mechanism as a possible explanation for the robustness and evolvability advantage of viruses evolved under low-moi conditions.

We sought to test whether the observed survival difference between robust and brittle viruses under moderately elevated temperatures carried over to a separate environmental perturbation known to cause virus damage and degradation. If so, the results would suggest that the genetically robust viruses are generally advantaged in terms of environmental robustness as well. Some theory predicts that genetic robustness and generalized environmental robustness should coincide, whereas other theory suggests no such relationship should be expected (de Visser et al. 2003). If equivalent survival of robust and brittle viruses was observed in an environmental perturbation other than thermal stress, it would further suggest that protein stability under heat shock may be the causal mechanism explaining robustness differences in phage $\phi 6$.

We chose survival under ultraviolet (UV) radiation as an additional challenge environment to examine relative survival of genetically robust and brittle phage strains. UV radiation can be a mutagen that increases the number of mutational errors occurring during DNA or RNA replication. However, our preliminary results (data not shown) found no difference in the frequency of reversion mutants in experiments where genetically marked phages were and were not exposed to UV-C (254-nm wavelength) radiation. Consistent with this observation, prior work shows that UV alone is not highly mutagenic to phage $\phi 6$, but that UV is effective at causing damage to $\phi 6$ virions presumably due to protein degradation (Lytle et al. 1993)

Using the same 12 phage strains (six robust, six brittle) for which we measured thermal reaction norms, we tested relative ability for viruses to survive exposure to UV-C $(254 \mathrm{~nm})$ radiation. Each strain was grown to a high-titer lysate as described above. Then, 200- $\mu \mathrm{L}$ aliquots containing a known initial quantity of virus $\left(N_{\mathrm{i}}\right)$ were randomly distributed among wells of a flat-bottomed polystyrene 96-well plate. A UV illuminator (Spectroline Long Life Filter) was placed over the 96-well plate, exposing the virus samples to UV-C emission. Plates were exposed to UV radiation for durations of $5,7.5,10$, or $15 \mathrm{~min}$. Following exposure, viruses in the individual wells were titered using standard plating procedures to estimate the post-UV exposure number of surviving virus particles $\left(N_{\mathrm{f}}\right) . \% S$ was calculated similarly to the temperature reaction-norm experiments $\left(\left[N_{\mathrm{f}} / N_{\mathrm{i}}\right] \times 100\right)$. Between three and six replicate measurements were obtained for each phage strain at each of the UV-exposure durations.

The results showed that the robust and brittle viruses were similarly damaged by UV-C radiation, presenting "death curves" characterized by decreasing grand mean survival following longer durations of UV exposure (Fig. 7). Although visual inspection suggested that grand mean $\% S$ was saturating with increasing UV exposure, for both groups of viruses, a simple linear regression provided an adequate fit to the data that was not improved through a quadratic model (robust strains: $P<0.001$; brittle strains: $P<0.001$ ). The observations (Fig. 7) hinted that robust strains may be advantaged in relative survival under inter-

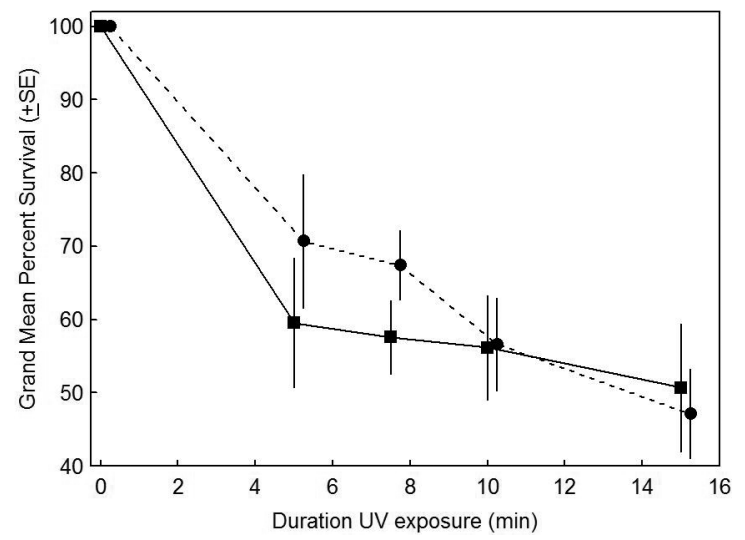

Figure 7. Strains of phage $\phi 6$ that differ in genetic robustness do not differ in survival following varying dosages of UV-C radiation. Robust (closed circles, dashed line) and brittle (closed squares, solid line) viruses were exposed to UV radiation for different durations, and the two groups of viruses showed equivalent survival as a function of UV dosage. Data points are offset for clarity.

mediate durations of UV exposure (7.5, $10 \mathrm{~min})$, echoing their greater survival at intermediate elevated temperatures. However, ANOVA showed that the interactive effect of treatment history (low- versus high-moi evolution) and duration of UV exposure on the dependent variable $\% S$ was not statistically significant $(P=0.686)$. Furthermore, a Kaplan-Meier estimator with a log-rank test did not demonstrate a significant difference in survival function for the two groups of viruses over the range of UV dosages examined $(P=0.674)$. Thus, our results did not suggest that genetically robust strains were relatively advantaged in survival under UV radiation, compared with their brittle counterparts.

Overall, the data hint that survival differences under moderate temperatures (but not under UV radiation) point to protein thermostability as a mechanism explaining relative differences in robustness among phage $\phi 6$ genotypes. Although survival of robust and brittle genotypes is equally poor at the elevated $45^{\circ} \mathrm{C}$ temperature, the general thermostability advantage of robust strains might have allowed lineages founded by these genotypes to more easily adapt when survival at $45^{\circ} \mathrm{C}$ was experienced as a selection pressure (McBride et al. 2008). These observations are consistent with the idea that robust organisms can accumulate genetic variation that is neutral in the current environment but which can be used for adaptive innovation when the environment changes. Thus, our work suggests that robust strains of the virus may be preadapted to better survive temperatures approaching (but not including) $45^{\circ} \mathrm{C}$, provided survival is predicated through adaptive change via natural selection rather than simply immediate survival upon entering the novel environment. We have cautioned that this observed adaptive advantage of robust genotypes may not generally carry over to other environmental challenges, such as viral adaptation to a novel host type that is important in virus emergence (McBride et al. 2008). Such generalized evolvability questions may be addressed using phage $\phi 6$ or other experi- 
mental systems where relative differences in robustness among genotypes have been observed.

\section{CONCLUDING REMARKS}

Our studies with phage $\phi 6$ provided a unique collection of virus genotypes that were shown to differ in their evolved genetic robustness (Montville et al. 2005). With this known difference in hand, we were able to capitalize on the collection of viruses to test whether a positive relationship exists between robustness and evolvability, as suggested by theory but rarely examined experimentally (McBride et al. 2008). This combination of rapidly evolving study system, observed differences in robustness, and existing theory on robustness and evolvability was admittedly fortuitous. Undoubtedly, these circumstances aided our ability to harness a biological system to test a hypothesis that predicts the course of future evolutionary events.

Traits other than genetic robustness, however, have been suggested to influence evolvability. One example is the presence of tandem repeats in promoter sequences of the yeast Saccharomyces cerevisiae, which are associated with higher rates of transcriptional divergence that indicate increased evolvability (Vinces et al. 2009). Other traits that have been associated with evolvability include variation in endocrine signaling pathways in social insects (Amdam et al. 2007) and protein dynamism (Tokuriki and Tawfik 2009). In addition, the molecular chaperone Hsp90 has been identified as a possible regulator of evolvability (Rutherford and Lindquist 1998) as has the yeast prion PSI (True and Lindquist 2000). These various biological systems could be harnessed to examine the proposed link between robustness and evolvability, in ways similar to our approach using phage $\phi 6$.

In addition, avoidance of extinction is a concept that relates to the propensity to adapt. Some studies have examined whether lineages or groups of organisms tend to avoid extinction better than others. A well-known result used fossil evidence to conclude that increased geographic range (i.e., niche breadth) decreased extinction probability in species of marine bivalves and gastropods (Jablonski 1986). This study supports the idea that greater habitat use should increase the likelihood that a species locates a habitat suitable for survival. One intriguing possibility for future research is to examine whether niche breadth in organisms such as pathogenic microbes similarly promotes avoidance of extinction. Resource generalization is often assumed to be costly, fostering arguments that natural species biodiversity can be explained by a tendency for niche specialists to be favored over niche generalists (Finke and Snyder 2008). However, many experimental evolution studies in microbes such as RNA viruses have shown that genotypes that evolve to use multiple hosts are not necessarily fitness disadvantaged relative to their more specialized counterparts (Novella et al. 1999; Turner and Elena 2000; Remold et al. 2008). These collections of microbes could be harnessed to conduct studies examining whether an evolved broad host range is superior to a narrow host range, when avoidance of extinction is the selective challenge.
Would the full elucidation of mechanisms for evolvability serve as a "Rosetta stone" for predictive evolution? Perhaps - but the very real challenge is to identify the myriad ways that an evolvability advantage might be obtained by biological systems. As suggested in studies exploring robustness and epistasis (Elena et al. 2006; Sanjuán and Elena 2006), it is quite possible that evolvability is governed by principles that are highly contextual and organism specific. That said, our proven ability to identify evolved robustness as a distinguishing trait in phage $\phi 6$, and to successfully use this knowledge to connect the trait to predictions regarding evolvability, reveals that such efforts are far from hopeless and that further experiments are warranted. If evolution can be truly transformed into a predictive science with a falsifiable set of governing principles, the implications would be enormous for basic research as well as biological applications. For example, public health would benefit by our increased ability to harness evolutionary predictions to prepare against emerging pathogens, and pharmacologists could be better informed in designing therapies that are resilient against evolutionary escape by targeted infectious agents. Overall, evolutionary biology could experience its next great paradigm shift, where the science becomes transformed from a discipline mostly concerned with elucidating the past to one that also boldly makes predictions about biological events that have yet to transpire.

\section{ACKNOWLEDGMENTS}

We thank Jeremy Draghi, James Pease, and Sonia Singhal for assistance with some of the experiments. This work was partially funded by a graduate dissertation fellowship to C.B.O. from UNCF-Merck, by National Institutes of Health training grant T32GM07205, and by grant DEB-0452163 to P.E.T. from the National Science Foundation.

\section{REFERENCES}

Amdam GV, Nilsen KA, Norberg K, Fondrk MK, Hartfelder K. 2007. Variation in endocrine signaling underlies variation in social life history. Am Nat 170: 37-46.

Bloom JD, Arnold FH. 2009. In the light of directed evolution: Pathways of adaptive protein evolution. Proc Natl Acad Sci (suppl. 1) 106: 9995-10000.

Bloom JD, Wilke CO, Arnold FH, Adami C. 2004. Stability and the evolvability of function in a model protein. Biophys $J$ 86: 2758-2764.

Bloom JD, Labthavikul ST, Otey CR, Arnold FH. 2006. Protein stability promotes evolvability. Proc Natl Acad Sci 103: 5869-5874.

Bloom JD, Lu Z, Chen D, Raval A, Venturelli OS, Arnold FH. 2007. Evolution favors protein mutational robustness in sufficiently large populations. BMC Biol 5: 29.

Brookfield JF. 2009. Evolution and evolvability: Celebrating Darwin 200. Biol Lett 5: 44-46.

Bornberg-Bauer E. 1997. How are model protein structures distributed in sequence space? Biophys J 73: 2393-2403.

Bornberg-Bauer E, Chan HS. 1999. Modeling evolutionary landscapes: Mutational stability, topology, and superfunnels in sequence space. Proc Natl Acad Sci 96: 10689-10694.

Burch CL, Chao L. 2004. Epistasis and its relationship to canalization in the RNA virus $\phi 6$. Genetics 167: 559-567.

Bush RM, Bender CA, Subbarao K, Cox NJ, Fitch WM. 1999. 
Predicting the evolution of human influenza A. Science 286: 1921-1925.

Chao L. 1990. Fitness of RNA virus decreased by Muller's ratchet. Nature 348: 454-455.

Coburn BJ, Wagner BG, Blower S. 2009. Modeling influenza epidemics and pandemics: Insights into the future of swine flu (H1N1). BMC Med 7: 30.

Codoñer FM, Darós JA, Solé RV, Elena SF. 2006. The fittest versus the flattest: Experimental confirmation of the quasispecies effect with subviral pathogens. PLoS Pathog 2:e136.

Craig EA, Gambill BD, Nelson RJ. 1993. Heat shock proteins: Molecular chaperones of protein biogenesis. Microbiol Rev 57: 402-414.

Cressman R, Krivan V, Garay J. 2004. Ideal free distributions, evolutionary games, and population dynamics in multiplespecies environments. Am Nat 164: 473-489.

Darwin C. 1859. On the origin of species by means of natural selection, or the preservation of favoured races in the struggle for life. Murray, London.

de Visser JA, Hermisson J, Wagner GP, Ancel Meyers L, Bagheri-Chaichian H, Blanchard JL, Chao L, Cheverud JM, Elena SF, Fontana W, et al. 2003. Perspective: Evolution and detection of genetic robustness. Evolution 57: 1959-1972.

Drake JW, Charlesworth B, Charlesworth D, Crow JF. 1998. Rates of spontaneous mutation. Genetics 148: 1667-1686.

Elena SF, Carrasco P, Daròs JA, Sanjuán R. 2006. Mechanisms of genetic robustness in RNA viruses. EMBO Rep 7: 168-173.

England JL, Shakhnovich BE, Shakhnovich EI. 2003. Natural selection of more designable folds: A mechanism for thermophilic adaptation. Proc Natl Acad Sci 100: 8727-8731.

Finke DL, Snyder WE. 2008. Niche partitioning increases resource exploitation by diverse communities. Science 321: 1488-1490.

Froissart R, Wilke CO, Montville R, Remold SK, Chao L, Turner PE. 2004. Co-infection weakens selection against epistatic mutations in RNA viruses. Genetics 168: 9-19.

Huber R, Stetter KO. 2001. Discovery of hyperthermophilic microorganisms. Methods Enzymol 330: 11-24.

Jablonski D. 1986. Background and mass extinctions: The alternation of macroevolutionary regimes. Science 231: 129-133.

Kirschner M, Gerhart J. 1998. Evolvability. Proc Natl Acad Sci 95: 8420-8427.

Koelle K, Cobey S, Grenfell B, Pascual M. 2006. Epochal evolution shapes the phylodynamics of interpandemic influenza A (H3N2) in humans. Science 314: 1898-1903.

Kumar S, Tsai CJ, Nussinov R. 2001. Thermodynamic differences among homologous thermophilic and mesophilic proteins. Biochemistry 40: 14152-14165.

Lenski RE, Travisano M. 1994. Dynamics of adaptation and diversification: A 10,000-generation experiment with bacterial populations. Proc Natl Acad Sci 91: 6808-6814.

Lenski RE, Ofria C, Pennock RT, Adami C. 2003. The evolutionary origin of complex features. Nature 423: 139-144.

Lytle CD, Wagner SJ, Prodouz NJ. 1993. Antiviral activity of gilvocarcin V plus UVA radiation. Photochem Photobiol 58: 818-821.

Maynard Smith J. 1982. Evolution and the theory of games. Cambridge University Press, Cambridge.

McBride RC, Turner PE. 2008. Genetic robustness and adaptability of viruses. Microbe 3: 409-415.

McBride RC, Ogbunugafor CB, Turner PE. 2008. Robustness promotes evolvability of thermotolerance in an RNA virus. BMC Evol Biol 8: 231

Montville R, Froissart R, Remold SK, Tenaillon O, Turner PE. 2005. Evolution of mutational robustness in an RNA virus. PLoS Biol 3: 1939-1945.

Novella IS, Hershey CL, Escarmis C, Domingo E, Holland JJ. 1999. Lack of evolutionary stasis during alternating replication of an arbovirus in insect and mammalian cells. J Mol Biol 287: 459-465.
Ortlund EA, Bridgham JT, Redinbo MR, Thornton RW. 2007. Crystal structure of an ancient protein: Evolution by conformational epistasis. Science 317: 1544-1548.

Parsell DA, Taulien J, Lindquist S. 1993. The role of heat-shock proteins in thermotolerance. Philos Trans R Soc Lond B Biol Sci 339: 279-285

Razvi A, Scholtz JM. 2006. Lessons in stability from thermophilic proteins. Protein Sci 15: 1569-1578.

Remold SK, Rambaut A, Turner PE. 2008. Evolutionary genomics of host adaptation in vesicular stomatitis virus. Mol Biol Evol 25: 1138-1147.

Rutherford SL, Lindquist S. 1998. Hsp90 as a capacitor for morphological evolution. Nature 396: 336-342

Sanjuán R, Elena SF. 2006. Epistasis correlates to genomic complexity. Proc Natl Acad Sci 103: 14402-14405.

Sanjuán R, Cuevas JM, Furió V, Holmes EC, Moya A. 2007. Selection for robustness in mutagenized RNA viruses. PLoS Genet 3: 939-946.

Silander OK, Weinreich DM, Wright KM, O'Keefe KJ, Rang CU, Turner PE, Chao L. 2005. Widespread genetic exchange among terrestrial bacteriophages. Proc Natl Acad Sci 102: 19009-19014.

Sterner R, Liebl W. 2001. Thermophilic adaptation of proteins. Crit Rev Biochem Mol Biol 36: 39-106.

Tanay A, Regev A, Shamir R. 2005. Conservation and evolvability in regulatory networks: The evolution of ribosomal regulation in yeast. Proc Natl Acad Sci 102: 7203-7208.

Thornton JW, Need E, Crews D. 2003. Resurrecting the ancestral steroid receptor: Ancient origin of estrogen signaling. Science 301: 1714-1717.

Tokuriki N, Tawfik DS. 2009. Protein dynamism and evolvability. Science 324: 203-207.

True HL, Lindquist SL. 2000. A yeast prion provides a mechanism for genetic variation and phenotypic diversity. Nature 407: 477-483.

Turner PE, Chao L. 1998. Sex and the evolution of intrahost competition in RNA virus $\phi 6$. Genetics 150: 523-532.

Turner PE, Chao L. 1999. Prisoner's dilemma in an RNA virus. Nature 398: 441-443.

Turner PE, Chao L. 2003. Escape from prisoner's dilemma in RNA phage $\phi 6$. Am Nat 161: 497-505.

Turner PE, Elena SF. 2000. Cost of host radiation in an RNA virus. Genetics 156: 1465-1470.

Turner PE, Burch CL, Hanley KA, Chao L. 1999. Hybrid frequencies confirm limit to coinfection in the RNA bacteriophage $\phi 6 . J$ Virol 73: 2420-2424.

Vinces MD, Legendre M, Caldara M, Hagihara M, Verstrepen KJ. 2009. Unstable tandem repeats in promoters confer transcriptional evolvability. Science 324: 1213-1216.

Vrba ES, DeGusta D. 2004. Do species populations really start small? New perspectives from the Late Neogene fossil record of African mammals. Philos Trans R Soc Lond B Biol Sci 359: 285-293.

Wagner A. 2005. Robustness, evolvability, and neutrality. FEBS Lett 579: 1772-1778.

Wagner A. 2006. Cooperation is fleeting in the world of transposable elements. PLoS Comput Biol 2: e162.

Wagner GP, Altenberg L. 1996. Complex adaptations and the evolution of evolvability. Evolution 50: 967-976.

Wagner GP, Booth G, Bagheri-Chaichian H. 1997. A population genetic theory of canalization. Evolution 51: 329-347.

Weinreich DM, Delaney NF, DePristo MA, Hartl DL. 2006. Darwinian evolution can follow only very few mutational paths to fitter proteins. Science 312: 111-114.

Wilke CO, Adami C. 2003. Evolution of mutational robustness. Mutat Res 522: 3-11.

Wilke CO, Wang JL, Ofria C, Lenski RE, Adami C. 2001. Evolution of digital organisms at high mutation rates leads to survival of the flattest. Nature 412: 331-333. 


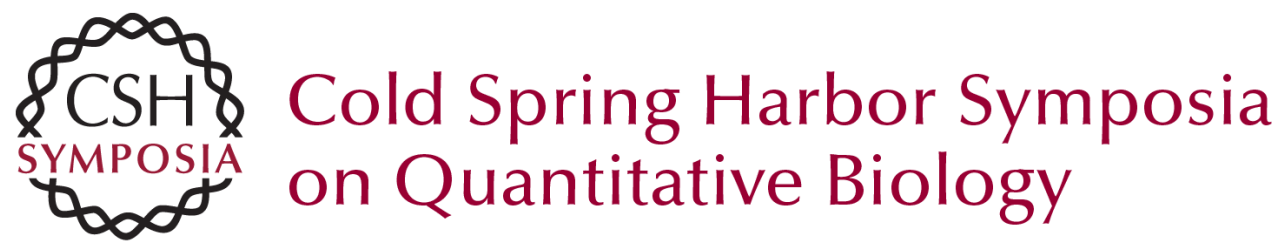

\title{
Predicting Virus Evolution: The Relationship between Genetic Robustness and Evolvability of Thermotolerance
}

\author{
C.B. Ogbunugafor, R.C. McBride and P.E. Turner
}

Cold Spring Harb Symp Quant Biol 2009 74: 109-118 originally published online October 20, 2009 Access the most recent version at doi:10.1101/sqb.2009.74.023

References This article cites 60 articles, 20 of which can be accessed free at: http://symposium.cshlp.org/content/74/109.full.html\#ref-list-1

\section{License}

Email Alerting

Receive free email alerts when new articles cite this article - sign up in the box at the Service top right corner of the article or click here.

To subscribe to Cold Spring Harbor Symposia on Quantitative Biology go to: http://symposium.cshlp.org/subscriptions 